\title{
Concomitant H1N1 encephalopathy and acute ischemic stroke: A case report
}

\author{
Elzbieta Wirkowski, Jay Yasen, Christopher Bondoc
}

Medical Director of NeuroICU, Winthrop University Hospital, New York, USA

Email: ewirkows@winthrop.org

Received 19 November 2011; revised 24 December 2011; accepted 5 January 2012

\begin{abstract}
Neurological complications of infection by the novel H1N1 virus have been described primarily in children. In adults, patients typically present with fever and respiratory symptoms, and may develop acute respiratory distress syndrome, sepsis and stroke. We present a 45-year-old man whose presentation of aphasia and right hemiplegia were incongruent with the findings on MRI of a small acute ischemic stroke. He rapidly developed multi-organ failure and died. Nasal swab PCR obtained prior to death returned positive for influenza virus $A / H 1 N 1$. At autopsy, a small acute hemorrhagic infarction in the left parietal lobe was present. We suspect that this was a cardio-embolic stroke, probably triggered by H1N1 myocarditis.
\end{abstract}

Keywords: Stroke; Infection

\section{CASE}

In December 2009, a 45-year-old man was awakened by his wife and was unable to speak. He fell while trying to get out of bed, and he had right sided weakness and incontinence of urine.

His past medical history was significant for hypertension, diabetes mellitus, chronic renal insufficiency, and congestive heart failure due to a non-ischemic cardiomyopathy since age 19 . The etiology of the cardiomyopathy was unknown. A recent ejection fraction was $40 \%$. He also had an anterior cervical discectomy and interbody fusion at C3-4 one month prior to admission. Medications included daily enalapril, atenolol, lasix, digoxin, insulin, simvastatin, and neurontin. He had no known allergies. There was no history of alcohol, tobacco, or illicit drug use. Family history was notable for diabetes mellitus.

On examination, he was afebrile. The pulse was 103 beats per minute, the blood pressure 130/70 mm Hg, the respiratory rate 16 beats per minute, and oxygen saturation $100 \%$. Blood sugar was $267 \mathrm{mg} / \mathrm{dL}$. Neck was sup- ple. The remainder of the general examination was normal. On neurological exam, he was awake with right hemineglect and global aphasia. There was no definite visual field loss, and no gaze deviation. Pupils were equal, round, and reactive to light. There was a right central facial nerve palsy and a flaccid right hemiplegia. Sensory testing was unreliable.

Admission laboratory values were notable for a blood urea nitrogen level of $35 \mathrm{mg}$ per deciliter, creatinine level of $1.5 \mathrm{mg} / \mathrm{dL}$, INR of 1.6 and CPK of $296 \mathrm{IU} / \mathrm{L}$ with troponin initially normal than increased to 4.8 $\mathrm{ng} / \mathrm{mL}$. Urine toxicology was normal. EKG showed sinus tachycardia with left atrial enlargement, and a left bundle branch block. Chest X-ray showed mild pulmonary venous congestion. Admission Head CT showed a small acute infarct in the left posterior parieto-occipital region, which was confirmed on the MRI of the brain (Figure 1). MRA of the brain and neck showed no significant stenosis. Transthoracic echocardiography on admission revealed a severely dilated left ventricle with an ejection fraction of less than $15 \%$. There were multiple highly mobile densities seen at the apex consistent with thrombi. There was also evidence of severe mitral regurgitation and moderate pulmonary hypertension.

The patient was admitted to the Neuro ICU and treated with intravenous phenytoin and heparin. Continuous videoEEG monitoring showed only generalized background slowing.

Because the MRI findings did not account for the clinical presentation of aphasia and right hemiplegia, a CT Perfusion was performed to evaluate for left cerebral hypoperfusion. There was global hypoperfusion without asymmetry, but it was a limited study due to poor intravenous contrast. On hospital day two, the patient was intubated and treated with a continuous infusion of Dobutamine in an attempt to improve brain and heart perfusion. Comprehension and right hemineglect improved. However, the right hemiplegia persisted. Followup imaging with Head CT showed no change. On hospital day three, the patient developed fever, and his condition deteriorated. Cultures of blood, sputum and urine 
were sent, and he was started on broad spectrum antibiotics and Acyclovir. Multiple cultures were negative. Repeat transthoracic echocardiography showed a further worsening. Twenty-four hour Holter monitor showed an intraventricular conduction defect and occasional multiform preventricular contractions with couplets. On hospital day four, the patient developed severe hypotension not responding to pressors, and multiorgan failure. On hospital day six, the patient had a cardiopulmonary arrest and expired. An autopsy revealed a small area of acute hemorrhagic infarct with no other pathology (Figure 2).

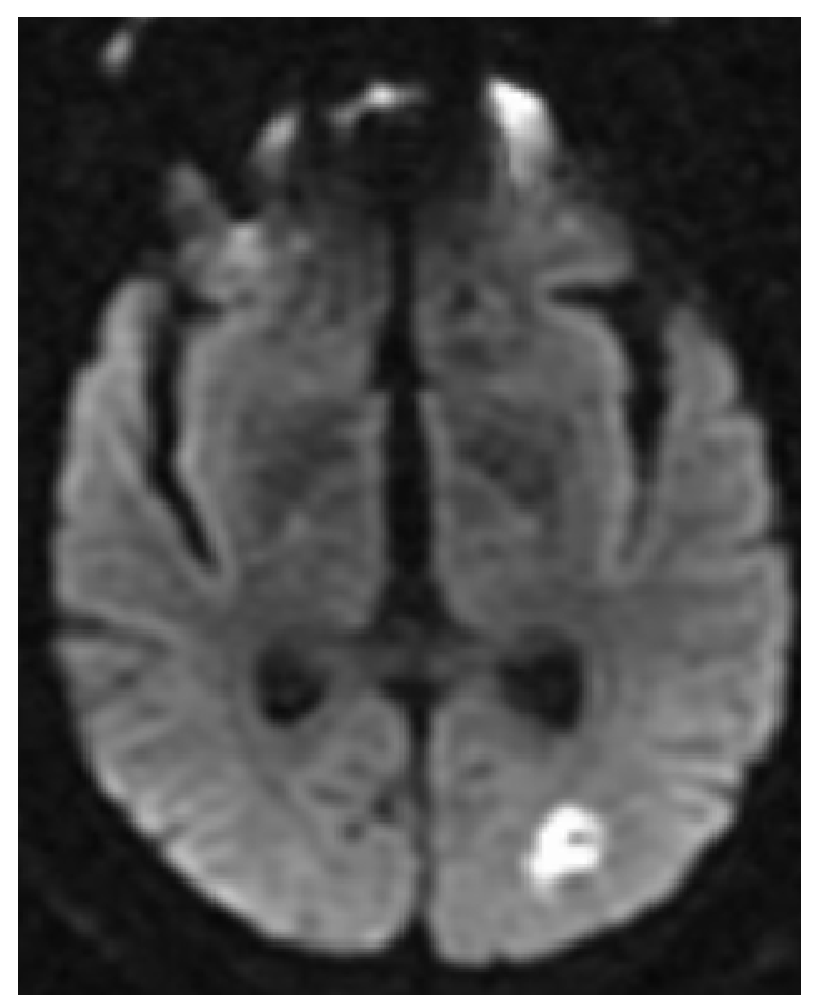

Figure 1. MRI brain (diffusion weighted imaging) showing restricted diffusion consistent with an acute infarct in the left posterior parieto-occipital region.

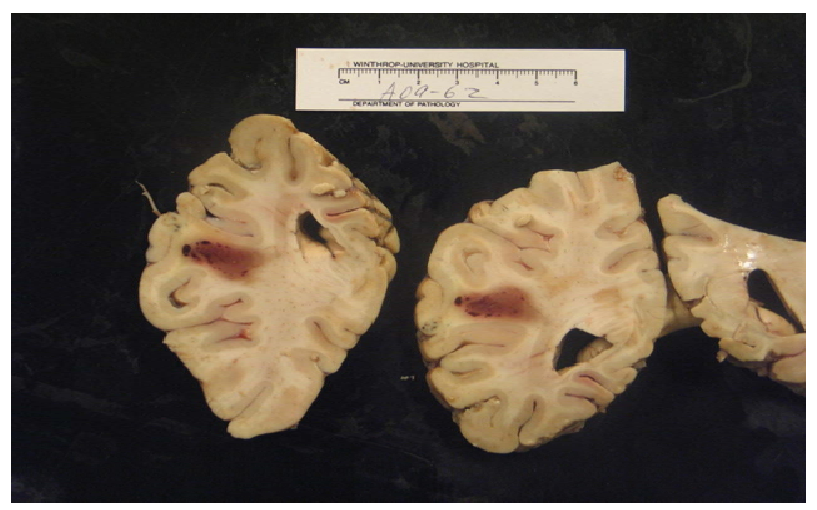

Figure 2. Autopsy revealed small area of acute hemorrhagic infarct.
The cerebral arteries showed no evidence of vasculitis, infection or inflammation (Figure 3). The day after the patient died a nasal swab which had been sent prior to death returned positive for influenza A/H1N1 by PCR.

\section{DISCUSSION}

Our patient's death was due to H1N1 infection and not due to stroke. H1N1 infection likely contributed to exacerbation of a preexisting cardiomyopathy, leading to both a low flow state as well as thrombo-emboli. However, the presentation of aphasia and right hemiplegia is not explained by the small left parieto-occipital infarct. It is also not explained by the global hypoperfusion demonstrated by the CT perfusion study, therefore infectious etiology is important. Non-convulsive seizures were excluded by continuous video-EEG monitoring. Fever, initially thought to be due to aspiration, not H1N1, may have contributed to the change in mental status. We suspect that the aphasia and right hemiplegia may have been due to H1N1 encephalopathy, essentially mimicking a larger stroke than the one which was actually present. The mechanism of this encephalopathy is unclear, Global cerebral hypoperfusion may have played an important role, though, it would not account for the right hemiplegia without additional modifying factors.

The most common symptoms in H1N1 are fever and cough, followed by sore throat and shortness of breath. Other symptoms include headache, rhinorrhea, myalgias, arthralgias, fatigue, vomiting, and diarrhea. In severe cases, patients generally begin to deteriorate around 3 to 5 days after symptom onset. Deterioration is rapid, with many patients progress to respiratory failure within 24 hours, requiring immediate admission to an intensive care unit.

Webster described cases in children or young adults in

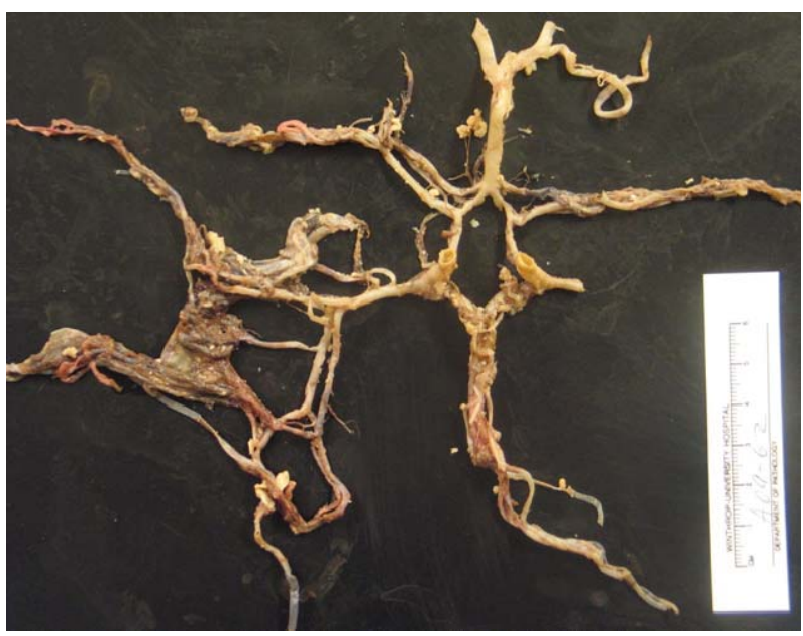

Figure 3. Cerebral arteries showed no evidence of vasculitis, infection or inflammation. 
which H1N1 virus presented as stroke like symptoms. In one case, a 5-year-old girl presented with 3 days of fever, right occipital headache, confusion, and drowsiness. She was febrile, agitated, and had a left hemiplegia. Brain MRI showed edema in the right parieto-occipital cortex on T2-weighted images with restricted diffusion in the underlying subcortical white matter. A nasopharyngeal swab specimen was positive for influenza H1N1.

In a second case, a 5-year-old boy developed fever, ascending paralysis, ophthalmoplegia, and drowsiness. Within 24 hours he was quadriplegic and comatose. [1]

In a report by Kitcharoen et al., 83 children were admitted with H1N1 virus infection to a tertiary children's hospital in a European setting between April 2009 and March 2010. Five children between the ages of 2 and 10 years had neurological symptoms. Four patients had seizures and encephalopathy at presentation. One patient presented with ataxia; one developed neuropsychiatric manifestations, and two developed movement disorders. Neuroimaging showed evidence of acute necrotizing encephalopathy in one case and non-specific white matter changes in another. Clinical outcomes varied: two recovered fully, while three had residual seizures and/or significant cognitive deficits [2].

Although the majority of neurological complications have been described in children, there are several cases described in adults:

Tan et al. described a previously healthy 34-year-old man who was admitted with influenza-like symptoms. Two days after admission, progressive quadriparesis with bilateral, symmetric paresthesias (glove-and-stocking pattern), and areflexia developed. His motor weakness (grade III/V) began in both legs, and then involved both arms. A computed tomography scan of the brain showed diffuse white matter lesions. A nasopharyngeal aspirate specimen was positive for pandemic (H1N1) 2009 virus by PCR [3].

Pathophysiology of H1N1 encephalopathy is likely a parainfectious, immune mediated process. Direct virulence of the virus is not implicated. The virus has not been identified at autopsy. This is consistent with our case, in which virus was also not identified in the brain tissue at autopsy.

Establishing a diagnosis of 2009 pandemic influenza A (H1N1) virus infection in hospitalized patients can be challenging, especially in patients presenting late in their clinical course. Although real-time reverse-transcriptase polymerase chain reaction (RT-PCR) is the most sensitive testing method to detect 2009 H1N1 virus in respiratory specimens, results are not immediately accessible [2]. That again was the case in our patient.

Stroke can be mimicked by many conditions. Encephalopathy, as it occurs in early sepsis, decompensated diabetes or hypertensive crises, can be confused with stroke. Encephalitis can initially be confused with stroke as well [4]. In particular, the acute onset of aphasia which sometimes occurs in Herpes Simplex Encephalitis is often initially confused with stroke. The presence of fever, typical MRI characteristics and CSF profile usually clarify the diagnosis. Seizures can lead to confusion with ischemic stroke, especially if non-convulsive seizures or a post-ictal deficit occurs.

We suspect that the worsening of our patient's preexisting cardiomyopathy was due to viral myocarditis. The most frequently implicated viruses in myocarditis are: Coxsackie B virus, hepatitis C, cytomegalovirus (CMV), echovirus, and influenza virus [5]. H1N1 influenza an infection is another cause of myocarditis, [5] and we suspect that this was the etiology of our patient's myocarditis. Impaired contractility and diminished wall motion contribute to cerebral hypoperfusion and lead to thrombo-embolic complications.

In summary, the presence of H1N1 infection contributed to a significant deterioration in cardiac function, leading to both perfusion failure as well as cerebral embolism. He died of sepsis secondary to H1N1. In addition, we speculate that his initial clinical presentation of aphasia and right hemiplegia may have been due to H1N1 encephalopathy, mimicking a stroke.

\section{REFERENCES}

[1] Webster, R.I., Hazelton, B., Suleiman, J., MaCartney, K., Kesson A. and Dale R.C. (2010) Infection in childhood: Case reports severe encephalopathy with swine origin influenza a H1N1. Neurology, 74, 1077-1078.

[2] Uyeki, T., Diagnostic testing for 2009 pandemic influenza a (H1N1) virus infection in hospitalized patients, The New England Journal of Medicine, 361, e114.

[3] Tan, K., Prerna, A. and Leo, Y.S. (2010) Surveillance of H1N1-related neurological complications. The Lancelet, 9, 142-143. doi:10.1016/S1474-4422(10)70015-6

[4] Libman, R. and Wirkowski, E. (1995) Conditions that mimic stroke in the emergency room, Archives of Neurology, 52, 1119-1122. doi:10.1001/archneur.1995.00540350113023

[5] Bowles, N.E. and Kearney, D.L. (2003) Detection of viruses in myocardial tissue by PCR, evidence of adenovirus as a common causeof myocarditis in children and adults. Journal of the American College of Cardiology, 42, 466- 472 doi:10.1016/S0735-1097(03)00648-X

[6] Kitcharoen, S., Pattapongsin, M., Sawanyawisuth, K., Vincent, A. and Tiamkao, S. (2010) Neurologic manifestations of pandemic (H1N1) virus infection. Emerging Infectious Diseases, 16, 569-570. doi:10.3201/eid1603.091699 